\title{
On the Finite Volume Element Method for Self-Adjoint Parabolic Integrodifferential Equations
}

\author{
Mohamed Bahaj ${ }^{1}$ and Anas Rachid ${ }^{2}$ \\ ${ }^{1}$ Department of Mathematics and Computing Science, Faculty of Sciences and Technology, Hassan 1st University, \\ BP 577 Settat, Morocco \\ ${ }^{2}$ École Nationale Suéprieure d’Arts et Métiers-Casablanca, Université Hassan II Mohammedia-Casablanca, \\ BP 150 Mohammedia, Morocco
}

Correspondence should be addressed to Anas Rachid; rachid.anas@gmail.com

Received 26 December 2012; Accepted 15 April 2013

Academic Editor: Mario Ohlberger

Copyright (C) 2013 M. Bahaj and A. Rachid. This is an open access article distributed under the Creative Commons Attribution License, which permits unrestricted use, distribution, and reproduction in any medium, provided the original work is properly cited.

Finite volume element schemes for non-self-adjoint parabolic integrodifferential equations are derived and stated. For the spatially discrete scheme, optimal-order error estimates in $L^{2}, H^{1}$, and $L^{p}, W^{1, p}$ norms for $2 \leq p<\infty$, are obtained. In this paper, we also study the lumped mass modification. Based on the Crank-Nicolson method, a time discretization scheme is discussed and related error estimates are derived.

\section{Introduction}

The main purpose of this paper is to study semidiscrete and full discrete finite volume element method (FVE) for parabolic integrodifferential equation of the form

$$
\begin{gathered}
u_{t}-\nabla \cdot(A(x, t) \nabla u)-\int_{0}^{t} \nabla \cdot(B(x, t, s) \nabla u(s)) d s \\
=f(x, t), \quad \text { in } \Omega \times(0, T], \\
u=0, \quad \text { on } \partial \Omega \times(0, T], \\
u(\cdot, 0)=u_{0}, \quad \text { in } \Omega,
\end{gathered}
$$

where $\Omega$ is a bounded domain in $\mathbb{R}^{d}, d=2,3$, with smooth boundary $\partial \Omega$, and $T<\infty$. Here $A(t)$, a non-selfadjoint second-order strongly elliptic, and $B(t, s)$, an arbitrary second-order linear partial differential operator, both with coefficients depending smoothly on $x$ and $t, f=f(x, t)$ and $u_{0}(x)$ are known functions, which are assumed to be smooth and satisfy certain compatibility conditions for $x \in$ $\Omega$ and $t=0$, so that (1) has a unique solution in certain Sobolev space. Problem (1) occurs in nonlocal reactive flows in porous media, viscoelasticity, and heat conduction through materials with memory.

Finite volume method is an important numerical tool for solving partial differential equations. It has been widely used in several engineering fields, such as fluid mechanics, heat and mass transfer, and petroleum engineering. The method can be formulated in the finite difference framework or in the Petrov-Galerkin framework. Usually, the former one is called finite volume method [1], marker and cell (MAC) method [2], or cell-centered method [3], and the latter one is called finite volume element method (FVE) [4-9], covolume method [10], or vertex-centered method $[11,12]$. We refer to the monographs $[13,14]$ for general presentation of these methods. The most important property of FVE method is that it can preserve the conservation laws (mass, momentum, and heat flux) on each control volume. This important property, combined with adequate accuracy and ease of implementation, has attracted more people to do research in this field.

Recently, the authors in $[8,15]$ studied FVE method for general self-adjoint elliptic problems. The authors in [16] presented and analyzed the semidiscrete and full discrete symmetric finite volume schemes for a class of parabolic problems. In $[6,7]$ the authors have studied FVE for one- and 
two-dimensional parabolic integrodifferential equations and have obtained an optimal-order estimate in the $L^{2}$-norm. The regularity required on the exact solution $u$ is $W^{3, p}$ for $p>1$ which is higher when compared to that for finite element methods.

The aim of this paper is to study the convergence of FVE discretization for a nonself-adjoint parabolic integrodifferential problem (1). Both spatially discrete scheme and discretein-time scheme are analyzed, and optimal error estimates in $L^{2}$ and $H^{1}$ norms are proved using only energy method. We also explore and generalize that idea to develop the lumped mass modification and $L^{p}$ estimates, $2 \leq p<\infty$. Our analysis avoids the use of semigroup theory, and the regularity requirement on the solution is the same of that of finite element method. Furthermore, based on the Crank-Nicolson method the fully discrete scheme is analyzed and the related optimal error estimates are established.

This paper is organized as follows. In Section 2, we introduce some notations and present some preliminary materials to be used later. The Ritz-Volterra projection to finite volume element spaces is introduced and related estimates are carried out in Section 3. In Section 4, we estimate the error of the finite volume element approximations derived in the previous section. In Section 5, the lumped mass is presented and optimal estimates in $L^{2}$ and $H^{1}$ norms are obtained Finally, the Crank-Nicolson scheme is studied in Section 6.

\section{Finite Volume Element Scheme}

In this section, we introduce some material which will be used repeatedly hareafter. Throughout this paper, $C$ (with or without index) denotes a generic positive constant which does not depend on the spatial and time discretization parameters $h$ and $k$, respectively.

2.1. Notations. We will use $\|\cdot\|_{m}$ and $|\cdot|_{m}$ (resp., $\|\cdot\|_{m, p}$ and $\left.|\cdot|_{m, p}\right)$ to denote the norm and seminorm of the Sobolev space $H^{m}(\Omega)$ (resp., $W^{m, p}(\Omega)$ ). The scalar product and norm in $L^{2}(\Omega)$ are denoted by $(\cdot, \cdot)$ and $\|\cdot\|$, respectively. Let $H_{1}^{0}(\Omega)$ be the standard Sobolev subspace of $H^{1}(\Omega)$ of functions vanishing on $\partial \Omega$.

The weak form of $(1)$ is used to find $u(\cdot, t):[0, T] \rightarrow$ $H_{0}^{1}(\Omega)$, such that

$$
\begin{gathered}
\left(u_{t}, v\right)+A(t ; u, v)+\int_{0}^{t} B(t, s ; u(s), v) d s \\
=(f, v), \quad \forall v \in H_{0}^{1}(\Omega), \\
u(0)=u_{0},
\end{gathered}
$$

where

$$
\begin{gathered}
A(t ; u, v)=\int_{\Omega} A(x, t) \nabla u \cdot \nabla v \\
B(t, s ; u(s), v)=\int_{\Omega} B(x, t, s) \nabla u(s) \cdot \nabla v .
\end{gathered}
$$

Let $\mathscr{T}_{h}$ be a decomposition of $\Omega$ into triangles (for the $2 \mathrm{D}$ case) or tetrahedral (for the $3 \mathrm{D}$ case) with $h=\max h_{K}$, where $h_{K}$ is the diameter of the element $K \in \mathscr{T}_{h}$.

In order to describe the FVE method for solving problem (1), we will introduce a dual partition $\mathscr{T}_{h}^{*}$ based upon the original partition $\mathscr{T}_{h}$ whose elements are called control volumes. We construct the control volumes in the same way as in $[7,17]$. Let $z_{K}$ be a point of $K \in \mathscr{T}_{h}$. In the 2D case, on each edge $e$ of $K$ a point $q_{e}$ is selected; then we connect $z_{K}$ with line segments to $q_{e}$; thus, partitioning $K$ into three quadrilaterals $K_{z}, z \in Z_{h}(K)$, where $Z_{h}(K)$ are the vertices of $K$. Then with each vertex $z \in Z_{h}=\cup_{K \in \mathscr{T}_{h}} Z_{h}(K)$ we associate a control volume $V_{z}$, which consists of the union of the subregions $K_{z}$, sharing the vertex $z$ (see Figure 1 ).

Similarly, in the 3D case, on each of the four faces $S_{i}, i=$ $1, \ldots, 4$, a point $q_{S_{i}}, i=1, \ldots, 4$, is selected, and on each of the six edges $e$ a point $q_{e}$ is selected. On each of the two faces $S_{1}$ and $S_{2}$ of $K$ sharing an edge $e$, we connect $q_{S_{i}}, i=1,2$, with $q_{e}$ and with $z_{K}$ by line segments, thus, partitioning $K$ into twelve tetrahedron $K_{z}, z \in Z_{h}(K)$ (see Figure 2). Then for $z \in Z_{h}$ the control volume $V_{z}$ consists of the union of the subregions $K_{z}$ sharing the vertex $z$. Thus, we finally obtain a group of control volumes covering the domain $\Omega$, which is called the dual partition $\mathscr{T}_{h}^{*}$ of the triangulation $\mathscr{T}_{h}$. We denote by $Z_{h}^{0}$ the set of interior vertices and $N_{h}=\# Z_{h}^{0}$. For a vertex $z_{i} \in$ $Z_{h}^{0}$, let $\Pi(i)$ be the index set of those vertices that along with $z_{i}$ are in some element of $T_{h}$ (Figure 2).

There are various ways to introduce a regular dual partition $\mathscr{T}_{h}^{*}$. In this paper, we will also use the construction of the control volumes in which we let $z_{K}$ be the barycenter of $K \in \mathscr{T}_{h}$. In the $2 \mathrm{D}$ case, we choose $q_{e}$ to be the midpoint of the edge $e$ (see Figure 3 ).

In the $3 \mathrm{D}$ case, we choose $q_{e}$ to be the midpoint of the edge $e$ and $q_{S_{i}}$ to be the barycenter of the face $S_{i}$ (Figure 4).

We call the partition $\mathscr{T}_{h}^{*}$ regular or quasiuniform, if there exists a positive $C>0$ such that

$$
C^{-1} h^{2} \leq \operatorname{meas}\left(V_{z}\right) \leq C h^{2}, \quad \forall V_{z} \in \mathscr{T}_{h}^{*} .
$$

If the finite element triangulation $\mathscr{T}_{h}$ is quasiuniform, that is, there exists a positive $C>0$ such that

$$
C^{-1} h^{2} \leq \operatorname{meas}(K) \leq C h^{2}, \quad \forall K \in \mathscr{T}_{h},
$$

then the dual partition $\mathscr{T}_{h}^{*}$ is also quasiuniform.

Based on the triangulation $T_{h}$, let $S_{h}$ be the standard conforming finite element space of piecewise linear functions, defined on the triangulation $T_{h}$ as follows:

$$
S_{h}=\left\{v \in \mathscr{C}(\Omega):\left.v\right|_{K} \text { is linear } \forall K \in T_{h} \text {, and }\left.v\right|_{\Gamma}=0\right\} .
$$

Let $I_{h}: \mathscr{C}(\Omega) \rightarrow S_{h}$ be the standard interpolation operators, such that

$$
I_{h} u=\sum_{z \in Z_{h}^{0}} v_{z}(t) \varphi_{z}(x), \quad \forall v \in S_{h},
$$

where $\left\{\varphi_{z}\right\}_{z \in Z_{h}^{0}}$ are the standard basis functions of $S_{h}$ and $v_{z}(t)=v(t ; z)$. 


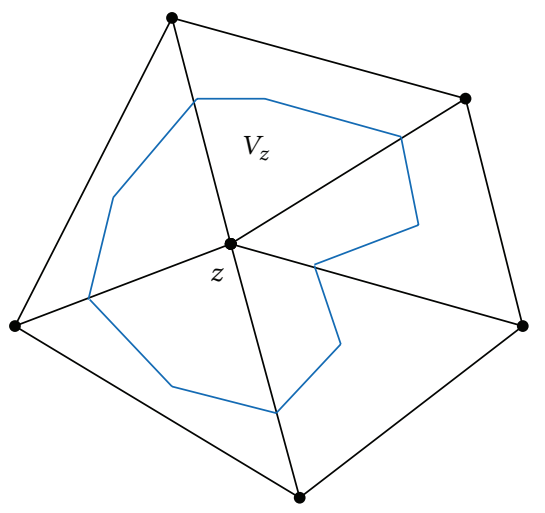

(a)

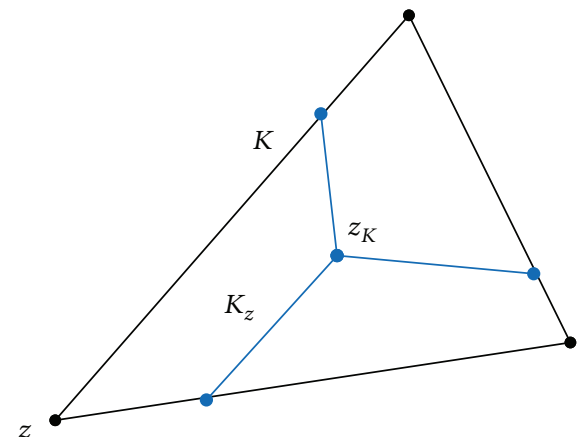

(b)

Figure 1: (a) A sample region with blue lines indicating the corresponding control volume $V_{z}$. (b) A triangle $K$ partitioned into three subregions $K_{z}$.

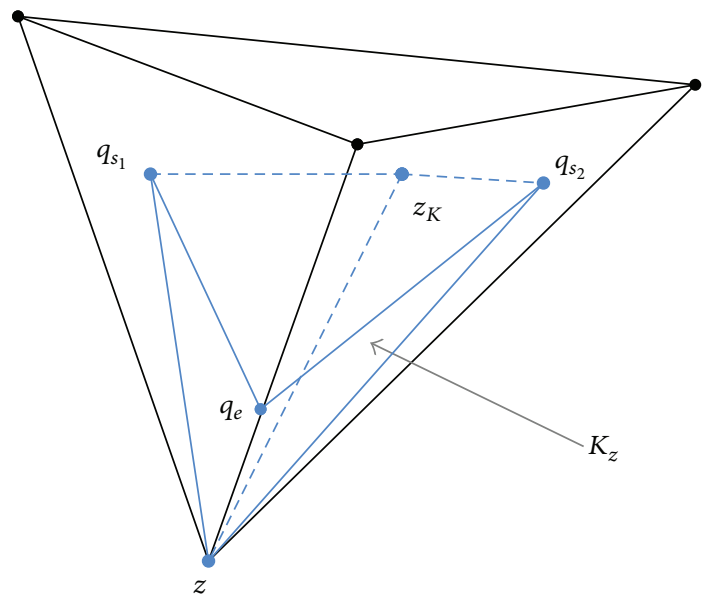

FIGURE 2: A tetrahedron $K$ partitioned into twelve subregions $K_{z}$.

2.2. Construction of the FVE Scheme. We formulate the FVE method for the problem (1) as follows: Given a $z \in Z_{h}^{0}$, integrating $(1)^{1}$ over the associated control volume $V_{z}$ and applying Green's formula, we obtain an integral conservation as follows form:

$$
\begin{aligned}
\int_{V_{z}} u_{t} & -\int_{\partial V_{z}} A(x, t) \nabla u \cdot n d s-\int_{\partial V_{z}} B(x, t, s) \nabla u \cdot n d s \\
& =\int_{V_{z}} f(x, t),
\end{aligned}
$$

where $n$ denotes the unit outer normal vector to $\partial V_{z}$.

Let $I_{h}^{*}: \mathscr{C}(\Omega) \rightarrow S_{h}^{*}$ be the transfer operator defined by

$$
I_{h}^{*} v=\sum_{z \in Z_{h}^{0}} v(z) \chi_{z}, \quad \forall v \in S_{h},
$$

where

$$
S_{h}^{*}=\left\{v \in L^{2}(\Omega):\left.v_{i}\right|_{V_{z}} \text { is constant, } \forall z \in Z_{h}^{0}\right\},
$$

and $\chi_{z}$ is the characteristic function of the control volume $V_{z}$.
Now for $t>0$ and for an arbitrary $I_{h}^{*} v$, we multiply (8) by $v(z)$, and sum over all $z \in Z_{h}^{0}$. Then the semidiscrete FVE approximation $u_{h}$ of (1) is a solution to the following problem: find $u_{h}(t) \in S_{h}$ for $t>0$ such that

$$
\begin{gathered}
\left(u_{h t}, v_{h}\right)+A\left(t ; u_{h}, v_{h}\right)+\int_{0}^{t} B\left(t, s ; u_{h}(s), v_{h}\right) d s \\
=\left(f, v_{h}\right) \quad v_{h} \in S_{h}^{*}, \\
u_{h}(0)=u_{0 h} \in S_{h} .
\end{gathered}
$$

Here the bilinear forms $A(t ; u, v)$ and $B(t, s ; u, v)$ are defined by

$$
\begin{aligned}
& A(t ; u, v) \\
& = \begin{cases}-\sum_{z \in Z_{h}^{0}} v_{i} \int_{\partial V z} A(x, t) \nabla u \cdot n d s, & (u, v) \in\left(\left(H_{0}^{1} \cap H^{2}\right) \cup S_{h}\right) \times S_{h}^{*}, \\
\int_{\Omega} A(x) \nabla u \cdot \nabla v d x, & (u, v) \in H_{0}^{1} \times H_{0}^{1},\end{cases} \\
& B(t, s ; u, v) \\
& = \begin{cases}-\sum_{z \in Z_{h}^{0}} v_{z} \int_{\partial V z} B(x, t, s) \nabla u \cdot n d s, & (u, v) \in\left(\left(H_{0}^{1} \cap H^{2}\right) \cup S_{h}\right) \times S_{h}^{*}, \\
\int_{\Omega} B(x, t, s) \nabla u \cdot \nabla v d x, & (u, v) \in H_{0}^{1} \times H_{0}^{1} .\end{cases}
\end{aligned}
$$

Let

$$
\begin{gathered}
u_{h}=\sum_{j=1}^{N_{h}} \alpha_{z}(t) \varphi_{z}(x), \\
\alpha(t)=\left(\alpha_{1}(t), \alpha_{2}(t), \ldots, \alpha_{N_{h}}(t)\right)^{T} .
\end{gathered}
$$

Then, we can rewrite scheme $(11)^{1}$ as systems of ordinary differential equations as follows:

$$
M_{h} \alpha^{\prime}(t)+A_{h}(t) \alpha(t)+\int_{0}^{t} B_{h}(t, s) \alpha(s) d s=F_{h}(t) .
$$




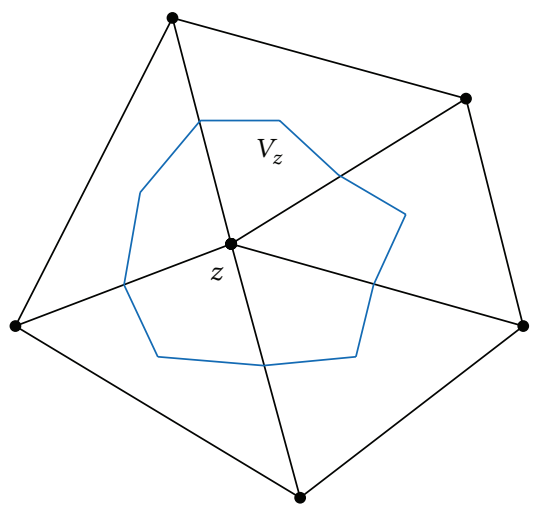

(a)

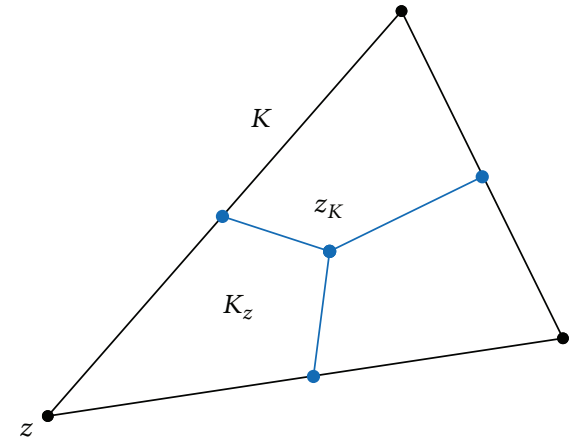

(b)

FIgURE 3: $z_{K}$ is the barycenter of $K$, and $q_{e}$ is to be the midpoint of the edge $e$.

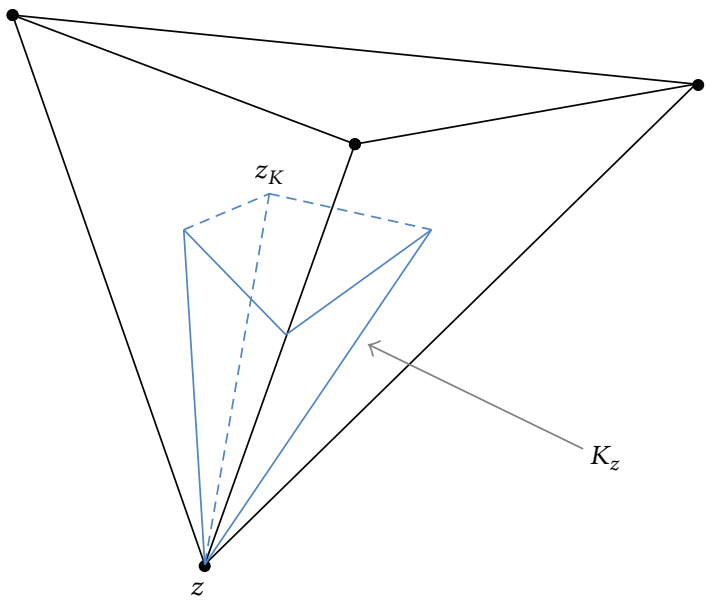

FIGURE 4: $q_{e}$ is the midpoint of the edge $e$, and $q_{S_{i}}$ is the barycenter of the face $S_{i}$.

Here $F_{h}(t)=\left(f_{1}(t), f_{2}(t), \ldots, f_{N_{h}}(t)\right)^{T}$, the mass matrix $M_{h}=\left\{M_{h_{i j}}\right\}=\left\{\left(\varphi_{i}, \chi_{j}\right)\right\}$ is tridiagonal, and both $A_{h}(t)=$ $\left\{A\left(t ; \varphi_{i}, \chi_{j}\right)\right\}$ and $B_{h}(t, s)=\left\{B\left(t, s ; \varphi_{i}, \chi_{j}\right)\right\}$ are positive definites.

In order to describe features of the bilinear forms defined in (11), we introduce some discrete norms on $S_{h}$ in the same way as in [7]:

$$
\begin{gathered}
\left\|v_{h}\right\|_{0, h}^{2}=\left(v_{h}, v_{h}\right)_{0, h}=\left(I_{h}^{*} v_{h}, I_{h}^{*} v_{h}\right), \\
\left|v_{h}\right|_{1, h}^{2}=\sum_{x_{i} \in Z_{h}^{0}} \sum_{x_{j} \in \Pi(i)} \operatorname{meas}\left(V_{i}\right)\left(\frac{v_{i}-v_{j}}{d_{i j}}\right)^{2}, \\
\left\|v_{h}\right\|_{1, h}^{2}=\left\|v_{h}\right\|_{0, h}^{2}+\left|v_{h}\right|_{1, h}^{2}, \quad\left\|v_{h}\right\|^{2}=\left(v_{h}, I_{h}^{*} v_{h}\right),
\end{gathered}
$$

where $d_{i j}=d\left(x_{i}, x_{j}\right)$, the distance between $x_{i}$ and $x_{j}$. Obviously, these norms are well defined for $v_{h} \in S_{h}^{*}$ as well and $\left\|v_{h}\right\|_{0, h}=\|\| v_{h} \| \mid$.

Hereafter we state the equivalence of discrete norms $\|\cdot\|_{0, h}$ and $\|\cdot\|_{1, h}$ with usual norms $\|\cdot\|$ and $\|\cdot\|_{1}$ on $S_{h}$, respectively.
Lemma 1 (see [7]). There exist two positive constants $C_{0}$ and $C_{1}$ such that for all $v_{h} \in S_{h}$, we have

$$
\begin{array}{ll}
C_{0}\left\|v_{h}\right\|_{0, h} \leq\left\|v_{h}\right\| \leq C_{1}\left\|v_{h}\right\|_{0, h}, & \forall v_{h} \in S_{h}, \\
C_{0}\|\| v_{h}\|\leq\| v_{h}\left\|\leq C_{1}\right\| v_{h} \|,, & \forall v_{h} \in S_{h}, \\
C_{0}\left\|v_{h}\right\|_{1, h} \leq\left\|v_{h}\right\|_{1} \leq C_{1}\left\|v_{h}\right\|_{1, h}, & \forall v_{h} \in S_{h} .
\end{array}
$$
$[7,18])$

Lemma 2 (see [7]). There exist two positive constants $C$ and $C_{0}$ such that for all $u_{h}, v_{h} \in S_{h}$, we have

$$
\begin{gathered}
A\left(u_{h}, I_{h}^{*} v_{h}\right) \leq C\left\|u_{h}\right\|_{1}\left\|v_{h}\right\|_{1}, \quad \forall u_{h}, v_{h} \in S_{h}, \\
A\left(v_{h}, I_{h}^{*} v_{h}\right) \geq C_{0}\left\|v_{h}\right\|_{1}^{2}, \quad \forall v_{h} \in S_{h} .
\end{gathered}
$$

The following lemmas are proved in $[3,7]$, which give the key feature of the bilinear forms in the FVE method.

Lemma 3 (see [3]). Assume that $\varphi \in W_{0}^{1, p}$. Then, one has

$$
\begin{aligned}
A\left(t ; \varphi, v_{h}\right)-A\left(t ; \varphi, I_{h}^{*} v_{h}\right) & \\
= & \sum_{K \in \tau_{h}} \int_{\partial K}(A(t) \nabla \varphi \cdot \mathbf{n})\left(v_{h}-I_{h}^{*} v_{h}\right) d s \\
& -\sum_{K \in \tau_{h}} \int_{K}(\nabla \cdot A(t) \nabla \varphi)\left(v_{h}-I_{h}^{*} v_{h}\right) d s, \quad \forall v_{h} \in S_{h} .
\end{aligned}
$$

The aforementioned identity holds true when $A(\cdot, \cdot)$ is replaced by $B(t, s ; \cdot, \cdot)$.

Lemma 4 (see [3]). Assume that $\varphi \in S_{h}$. Then, one has

$$
A(t ; \varphi, \chi)-A\left(t ; \varphi, I_{h}^{*} \chi\right) \leq C h|\varphi|_{1, p}|\chi|_{1, q} .
$$

Furthermore, for $\varphi \in W_{0}^{1, p} \cap W^{2, p}$, we have

$$
A(t ; \varphi, \chi)-A\left(t ; \varphi, I_{h}^{*} \chi\right) \leq C h\|\varphi\|_{2, p}\|\chi\|_{1, q} .
$$




\section{Ritz-Volterra Projection and Related Estimates}

Following [7, 19, 20], we define the Ritz-Volterra projection $V_{h}(t): H_{0}^{1} \rightarrow S_{h}$ as follows:

$A\left(t ; u-V_{h} u, I_{h}^{*} v_{h}\right)+\int_{0}^{t} B\left(t, s ; u(s)-V_{h} u(s), I_{h}^{*} v_{h}\right) d s=0$, $t>0, \quad \forall v_{h} \in S_{h}$.

This $V_{h}(t)$ is an elliptic projection with memory of $u$ into $S_{h}^{*}$. It is easy to see that (21) is actually a system of integral equations of Volterra type. In fact, if $V_{h}(t) u=$ $\sum_{j=1}^{N_{h}} \alpha_{j}(t) \varphi_{j}(x)$, then (21) can be rewritten as

$$
A_{h}(t) \alpha(t)+\int_{0}^{t} B_{h}(t, s) \alpha(s) d s=F_{h}(t)
$$

where $A_{h}(t), B_{h}(t, s)$ are matrices and $\alpha(t), F_{h}(t)$ are vectors, defined via

$$
\begin{gathered}
\alpha(t)=\left(\alpha_{1}(t), \alpha_{2}(t), \ldots, \alpha_{N_{h}}(t)\right)^{T}, \\
F_{h k}(t)=A\left(t ; u, \chi_{k}\right)+\int_{0}^{t} B\left(t, s ; u(s), \chi_{k}\right) d s \\
k=1,2, \ldots, N_{h}, \\
A_{h}(t)=A\left(t, \varphi_{k}(x), \chi_{l}\right), \quad B_{h}(t, s)=B\left(t, s ; \varphi_{k}(x), \chi_{l}\right) .
\end{gathered}
$$

From the positivity of $A$ (Lemma 2 ) and the linearity of (22), we see that the system (22) possesses a unique solution $\alpha(t)$. Consequently, $V_{h}(t) u$ in (21) is well defined.

Set $\rho=u-V_{h}(t) u$. The following lemma was proved in [7], which shows the $H^{1}$ error estimate for $\rho$ and its temporal derivative.

Lemma 5 (see [7]). Assume that $D_{t}^{n} u \in L^{\infty}\left(H_{0}^{1} \cap H^{2}\right)$ for all $0 \leq n \leq k$, for some integer $k \geq 0$. Then, for $T>0$ fixed there is a constant $C=C(T ; k)>0$, independent of $h$ and $u$, such that for all $0 \leq n \leq k$ and $0<t<T$,

$$
\begin{gathered}
\|\rho(t)\|_{1} \leq C h\left(\|u\|_{2}+\int_{0}^{t}\|u\|_{2} d s\right), \\
\left\|D_{t}^{n} \rho(t)\right\|_{1} \leq C h\left(\sum_{i=0}^{n}\left\|D_{t}^{i} u\right\|_{2}+\int_{0}^{t}\|u\|_{2} d s\right) .
\end{gathered}
$$

Now we establish $L^{2}$ error estimate for $\rho$ and its temporal derivative which improves Theorem 2.2 in [7]. This estimate is optimal with respect to the order.

Lemma 6. Assume that, for some integer $k \geq 0, D_{t}^{n} u \in$ $L^{\infty}\left(H_{0}^{1} \cap H^{2}\right)$ for all $0 \leq n \leq k$. Then, for $T>0$ fixed there is a constant $C=C(T ; k)>0$, independent of $h$ and $u$, such that for all $0 \leq n \leq k$ and $0<t<T$,

$$
\begin{gathered}
\|\rho(t)\| \leq C h^{2}\left(\|u\|_{2}+\int_{0}^{t}\|u\|_{2} d s\right), \\
\left\|D_{t}^{n} \rho(t)\right\| \leq C h^{2}\left(\sum_{i=0}^{n}\left\|D_{t}^{i} u\right\|_{2}+\int_{0}^{t}\|u\|_{2} d s\right) .
\end{gathered}
$$

Proof. The proof will proceed by duality argument. Let $\psi \in$ $H^{2}(\Omega) \cap H_{0}^{1}(\Omega)$ be the solution of

$$
\begin{gathered}
A^{*}(t) \psi=\rho, \quad \text { in } \Omega \\
\psi=0, \quad \text { in } \partial \Omega .
\end{gathered}
$$

The solution $\psi \in H^{2}(\Omega) \cap H_{0}^{1}(\Omega)$ satisfies the following regularity estimate:

$$
\|\psi\|_{2} \leq C\|\rho\|
$$

Multiplying this equation by $\rho$ and then taking $L^{2}$ innerproduct over $\Omega$, we obtain the following:

$$
\begin{aligned}
\|\rho\|^{2}= & A(t ; \rho, \psi) \\
= & A\left(t ; \rho, \psi-R_{h} \psi\right)+A\left(t ; \rho, R_{h} \psi-I_{h}^{*}\left(R_{h} \psi\right)\right) \\
& -\int_{0}^{t} B\left(t, s ; \rho(s), I_{h}^{*} R_{h} \psi-R_{h} \psi\right) d s \\
& -\int_{0}^{t} B\left(t, s ; \rho(s), R_{h} \psi-\psi\right) d s \\
& -\int_{0}^{t} B(t, s ; \rho(s), \psi) d s=I_{1}+I_{2}+I_{3}+I_{4}+I_{5} .
\end{aligned}
$$

We have

$$
\left|I_{1}\right|+\left|I_{4}\right| \leq C h^{2}\left(\|u\|_{2}+\int_{0}^{t}\|u\|_{2} d s\right)\|\psi\|_{2} .
$$

Applying Lemma 4, we obtain

$$
\left|I_{2}\right|+\left|I_{3}\right| \leq C h^{2}\left(\|u\|_{2}+\int_{0}^{t}\|u\|_{2} d s\right)\|\psi\|_{2} .
$$

Finally, we have

$$
\left|I_{5}\right| \leq \int_{0}^{t}\left(\rho(s), B^{*}(t, s) \psi\right) d s \leq C\left(\int_{0}^{t}\|\rho\| d s\right)\|\psi\|_{2} ;
$$

then, we have

$$
\|\rho\| \leq C h^{2}\left(\|u\|_{2}+\int_{0}^{t}\|u\|_{2} d s\right)+C\left(\int_{0}^{t}\|\rho\| d s\right) .
$$

Finally, an application of Gronwall's lemma yields the first estimate.

The second inequality follows in a similar fashion. 
Lemma 7. There exists a constant $C$ independent of $h$ such that

$$
\|\rho\|_{0, p}+h\|\rho\|_{1, p} \leq C h^{2}\left(\|u\|_{2, p}+\int_{0}^{t}\|u\|_{2, p} d s\right) .
$$

Proof. Let $\rho_{x}$ be an arbitrary component of $\nabla \rho$, with $p$ and $q$ conjugate indices; we have $\left\|\rho_{x}\right\|_{p}=\sup \left\{\left(\rho_{x}, \varphi\right) ; \varphi \in\right.$ $\left.\mathscr{C}_{0}^{\infty}(\Omega),\|\varphi\|_{q}=1\right\}$.

For any such $\varphi$, let $\psi$ be the solution of

$$
\begin{gathered}
A^{*}(t ; \psi, v)=-\left(\varphi_{x}, v\right) \quad \forall v \in H_{0}^{1}(\Omega), \\
\psi=0, \quad \text { on } \partial \Omega .
\end{gathered}
$$

It follows from the regularity theory for the elliptic problem that

$$
\|\psi\|_{1, q} \leq C_{p}\|\varphi\|_{q}=C_{p}
$$

We then have by application of (21) that

$$
\begin{aligned}
\left(\rho_{x}, \varphi\right)= & A(t ; \rho, \psi)=A\left(t ; \rho, \psi-R_{h} \psi\right) \\
& +A\left(t ; \rho, R_{h} \psi-I_{h}^{*}\left(R_{h} \psi\right)\right) \\
& +\int_{0}^{t} B\left(t, s ; \rho(s), I_{h}^{*}\left(R_{h} \psi\right)\right) d s \\
= & I_{1}+I_{2}+I_{3}, \\
A\left(t ; \rho, \psi-R_{h} \psi\right)= & A\left(t ; R_{h} u-u, \psi\right) \\
= & -\left(\left(R_{h} u-u\right)_{x}, \varphi\right) \leq C h\|u\|_{2, p} .
\end{aligned}
$$

Applying Lemma 4, we have

$$
\begin{aligned}
I_{2} & =A\left(t ; u, R_{h} \psi-I_{h}^{*}\left(R_{h} \psi\right)\right)-A\left(t ; V_{h} u, R_{h} \psi-I_{h}^{*}\left(R_{h} \psi\right)\right) \\
& \leq C h\|u\|_{2, p} .
\end{aligned}
$$

Finally, $I_{3}$ is estimated as follows:

$$
I_{3}=\int_{0}^{t} B\left(t, s ; \rho(s), I_{h}^{*}\left(R_{h} \psi\right)\right) d s \leq C_{p} \int_{0}^{t}\|\rho\|_{1, p} d s .
$$

Combining these estimates, we get

$$
\|\rho\|_{1, p} \leq C h\|u\|_{2, p}+C_{p} \int_{0}^{t}\|\rho\|_{1, p} d s
$$

hence by Gronwall's lemma

$$
\|\rho\|_{1, p} \leq C h\left(\|u\|_{2, p}+\int_{0}^{t}\|u\|_{2, p} d s\right) .
$$

The derivation of the error estimate in $L^{p}$ is similar to the case when $p=2$.

\section{Error Estimates for Semidiscrete Approximations}

We split the error $e(t)=u(t)-u_{h}(t)$ as follows:

$e(t)=\left(u(t)-V_{h} u(t)\right)+\left(V_{h} u(t)-u_{h}(t)\right)=\rho+\theta$.

It is easy to see that $\theta=V_{h} u(t)-u_{h}(t) \in S_{h}$ satisfies an error equation of the form

$$
\begin{gathered}
\left(\theta_{t}, I_{h}^{*} v_{h}\right)+A\left(t ; \theta, I_{h}^{*} v_{h}\right)+\int_{0}^{t} B\left(t, s ; \theta(s), I_{h}^{*} v_{h}\right) d s \\
\quad=-\left(\rho_{t}, I_{h}^{*} v_{h}\right), \quad v_{h} \in S_{h} .
\end{gathered}
$$

Since the estimates of $\rho$ are already known, it is enough to have estimates for $\theta$.

We will prove a sequence of lemmas which lead to the following result.

Lemma 8. There is a positive constant $C$ independent of $h$ such that

$$
\|\theta(t)\| \| \leq C\left(\|\mid \theta(0)\|\left\|^{2}+\int_{0}^{t}\right\| \rho_{t} \| d s\right) .
$$

Proof. Since $\theta \in S_{h}$ we may take $v_{h}=\theta$ in (42) to obtain

$$
\begin{aligned}
\frac{1}{2} \frac{d}{d t}\|\| \theta(t)\|\|^{2}+c\|\theta\|_{1}^{2} & \leq\left\|\rho_{t}\right\|\|\theta\|+C \int_{0}^{t}\|\theta\|_{1} d s\|\theta\|_{1} \\
& \leq\left\|\rho_{t}\right\|\|\theta\|+\frac{1}{2} c\|\theta\|_{1}^{2}+C \int_{0}^{t}\|\theta\|_{1}^{2} d s
\end{aligned}
$$

and hence by integration and Lemma 1, we have

$$
\begin{aligned}
& \|\theta(t)\|^{2}+\int_{0}^{t}\|\theta\|_{1}^{2} d s \\
& \quad \leq C\left(\|\theta(0)\|\left\|^{2}+\int_{0}^{t}\right\| \rho_{t}\|\| \theta\left\|d s+\int_{0}^{t} \int_{0}^{s}\right\| \theta(\tau) \|_{1}^{2} d \tau d s\right) .
\end{aligned}
$$

Gronwall's lemma now implies the following:

$$
\begin{aligned}
\|\| \theta(t)\|\|^{2}+\int_{0}^{t}\|\theta\|_{1}^{2} d s \leq & C\left(\|\theta(0)\|\left\|^{2}+\int_{0}^{t}\right\| \rho_{t}\|\| \theta \| d s\right) \\
\leq & C\|\| \theta(0)\|\|^{2}+\frac{1}{2} \sup _{s \leq t}\|\theta(s)\|^{2} \\
& +\left(\int_{0}^{t}\left\|\rho_{t}\right\| d s\right)^{2} .
\end{aligned}
$$

Since this holds for all $\in J$, we may conclude that

$$
\|\theta(t)\| \leq C\left(\|\theta(0)\|\left\|+\int_{0}^{t}\right\| \rho_{t} \| d s\right) .
$$


Remark 9. If the initial value was chosen so that $\left\|u_{0 h}-u_{0}\right\| \leq$ $C h^{2}\left\|u_{0}\right\|_{2}$, then $\|\theta(0)\| \leq\left\|u_{0 h}-u_{0}\right\|+\left\|V_{h} u_{0}-u_{0}\right\| \leq C h^{2}\left\|u_{0}\right\|_{2}$. One can derive

$$
\|\mid \theta(t)\| \| \leq C h^{2}\left(\left\|u_{0}\right\|_{2}+\int_{0}^{t}\left\|u_{t}\right\|_{2} d s\right) .
$$

Lemma 10. There is a positive constant $C$ independent of $h$ such that

$$
\int_{0}^{t}\left\|\theta_{t}\right\|^{2} d s+\|\theta\|_{1}^{2} \leq C\left(\|\theta(0)\|_{1}^{2}+\int_{0}^{t}\left\|\rho_{t}\right\|^{2} d s\right) .
$$

Proof. Set $v_{h}=\theta_{t}$ in (42) to get

$$
\begin{aligned}
\left\|\theta_{t}\right\|^{2}+ & \frac{1}{2} \frac{d}{d t} A\left(t ; \theta, I_{h}^{*} \theta\right) \\
= & -\left(\rho_{t}, I_{h}^{*} \theta_{t}\right)-\int_{0}^{t} B\left(t, s ; \theta(s), I_{h}^{*} \theta_{t}(t)\right) d s \\
& +\frac{1}{2} A_{t}\left(t ; \theta, I_{h}^{*} \theta\right) \\
& +\frac{1}{2}\left[A\left(t ; \theta_{t}, I_{h}^{*} \theta\right)-A\left(t ; \theta, I_{h}^{*} \theta_{t}\right)\right] \\
\leq & \frac{1}{2}\left\|\rho_{t}\right\|^{2}+\frac{1}{2}\|\| \theta_{t} \|^{2}+A_{t}\left(t ; \theta, I_{h}^{*} \theta\right) \\
& +\frac{1}{2}\left[A\left(t ; \theta_{t}, I_{h}^{*} \theta\right)-A\left(t ; \theta, I_{h}^{*} \theta_{t}\right)\right] \\
& -\frac{d}{d t} \int_{0}^{t} B\left(t, s ; \theta(s), I_{h}^{*} \theta(t)\right) d s \\
& +B\left(t, t ; \theta(t), I_{h}^{*} \theta(t)\right)+\int_{0}^{t} B_{t}\left(t, s ; \theta(s), I_{h}^{*} \theta(t)\right) d s .
\end{aligned}
$$

Then

$$
\begin{aligned}
\left\|\theta_{t}\right\|^{2}+ & \frac{d}{d t} A\left(t ; \theta, I_{h}^{*} \theta\right) \\
\leq & \left\|\rho_{t}\right\|^{2}-\frac{d}{d t} \int_{0}^{t} B\left(t, s ; \theta(s), I_{h}^{*} \theta\right) d s \\
& +C\left(\|\theta\|_{1}^{2}+\int_{0}^{t}\|\theta(s)\|_{1}^{2} d s\right) \\
& +\frac{1}{2}\left[A\left(t ; \theta_{t}, I_{h}^{*} \theta\right)-A\left(t ; \theta, I_{h}^{*} \theta_{t}\right)\right] .
\end{aligned}
$$

In addition, recall that

$$
\begin{array}{r}
A\left(t ; u_{h}, I_{h}^{*} v_{h}\right)-A\left(t ; v_{h}, I_{h}^{*} u_{h}\right) \leq C h\left\|u_{h}\right\|_{1}\left\|v_{h}\right\|_{1}, \\
\forall u_{h}, v_{h} \in S_{h} ;
\end{array}
$$

then applying an inverse inequality and using kickback argument, we obtain

$$
\begin{aligned}
{\left[A\left(t ; \theta_{t}, I_{h}^{*} \theta\right)-A\left(t ; \theta, I_{h}^{*} \theta_{t}\right)\right] } & \leq C h\left\|\theta_{t}\right\|_{1}\|\theta\|_{1} \leq C\left\|\theta_{t}\right\|\|\theta\|_{1} \\
& \leq \varepsilon\left\|\theta_{t}\right\|^{2}+C\|\theta\|_{1}^{2} .
\end{aligned}
$$

Combining these estimates, we derive

$$
\begin{aligned}
\left\|\theta_{t}\right\|^{2}+ & \frac{d}{d t} A\left(t ; \theta, I_{h}^{*} \theta\right) \\
\leq & \left\|\rho_{t}\right\|^{2}-\frac{d}{d t} \int_{0}^{t} B\left(t, s ; \theta(s), I_{h}^{*} \theta\right) d s \\
& +C\left(\|\theta\|_{1}^{2}+\int_{0}^{t}\|\theta(s)\|_{1}^{2} d s\right) .
\end{aligned}
$$

So after integration in time and using the weak coercivity of $A\left(t ; \theta, I_{h}^{*} \theta\right)$, we get

$$
\begin{aligned}
\int_{0}^{t}\left\|\theta_{t}\right\|^{2} d s+c_{0}\|\theta\|_{1}^{2} \\
\leq c_{0}\|\theta(0)\|_{1}^{2}+\int_{0}^{t}\left\|\rho_{t}\right\|^{2} d s \\
\quad+\int_{0}^{t} B\left(t, s ; \theta(s), I_{h}^{*} \theta\right) d s+C \int_{0}^{t}\|\theta(s)\|_{1}^{2} d s \\
\leq c_{0}\|\theta(0)\|_{1}^{2}+\frac{c}{2}\|\theta\|_{1}^{2}+C\left(\int_{0}^{t}\left\|\rho_{t}\right\|^{2}+\|\theta(s)\|_{1}^{2} d s\right),
\end{aligned}
$$

and by Gronwall's lemma,

$$
\int_{0}^{t}\left\|\theta_{t}\right\|^{2} d s+c\|\theta\|_{1}^{2} \leq C\left(\|\theta(0)\|_{1}^{2}+\int_{0}^{t}\left\|\rho_{t}\right\|^{2} d s\right) .
$$

Remark 11. If $\theta(0)=0$, then

$$
\int_{0}^{t}\left\|\theta_{t}\right\|^{2} d s+c\|\theta\|_{1}^{2} \leq C h^{2}\left(\int_{0}^{t}\left\|u_{t}\right\|_{2}^{2} d s\right) .
$$

Theorem 12 (error estimates in $L^{2}$ and $H^{1}$-norms). Let $u$, $u_{h}$ be the solutions of (2) and (11), respectively. Assume that $u, u_{t} \in$ $L^{\infty}\left(H_{0}^{1} \cap H^{2}\right)$.

(a) Let $u_{0 h}$ be chosen so that $\left\|u_{0 h}-u_{0}\right\| \leq C h^{2}\left\|u_{0}\right\|_{2}$. Then for $T>0$ fixed there is a constant $C=C(T)$ independent of $h$, such that for all $0<t<T$,

$$
\left\|u_{h}(t)-u(t)\right\| \leq C h^{2}\left(\left\|u_{0}\right\|_{2}+\int_{0}^{t}\left\|u_{t}\right\|_{2} d s\right) .
$$

(b) Let $u_{0 h}$ be chosen so that $\left\|u_{0 h}-u_{0}\right\|_{1} \leq C h\left\|u_{0}\right\|_{2}$. Then for $T>0$ fixed there is a constant $C=C(T)$ independent of $h$, such that for all $0<t<T$,

$$
\left.\| u_{h}(t)-u(t)\right) \|_{1} \leq C h\left(\left\|u_{0}\right\|_{2}+\int_{0}^{t}\left\|u_{t}\right\|_{2} d s\right) .
$$

We now prove error estimates for FVE approximations in $L^{p}$ and $W^{1, p}$-norms. 
Theorem 13 (error estimates in $L^{p}$ and $W^{1, p}$-norms). Let $u, u_{h}$ be the solutions of (2) and (11), respectively and $u_{0 h}=$ $V_{h} u_{0}$. Assume that $u, u_{t} \in L^{\infty}\left(H_{0}^{1} \cap W^{2, p}\right)$. For h sufficiently small, we have

$$
\begin{gathered}
\left\|u-u_{h}\right\|_{0, p} \leq C h^{2}\left(\left\|u_{0}\right\|_{2}+\int_{0}^{t}\left\|u_{t}\right\|_{2} d s\right), \\
\left\|u-u_{h}\right\|_{1, p} \leq C h\left(\left\|u_{0}\right\|_{2}+\|u\|_{2, p}+\int_{0}^{t}\left\|u_{t}\right\|_{2} d s\right) .
\end{gathered}
$$

Proof. If $2 \leq p<\infty$, by the following Sobolev embedding inequality

$$
\|\theta\|_{0, p} \leq C\|\theta\|_{1}
$$

then the first desired estimate follows from Lemmas 7 and 10.

Given $\varphi \in \mathscr{C}_{0}^{\infty}(\Omega)$, find $\psi \in H_{0}^{1}(\Omega)$ such that

$$
\begin{gathered}
A(t)^{*} \psi=-\varphi_{x}, \quad \text { in } \Omega, \\
\psi=0, \quad \text { on } \partial \Omega, \\
\|\psi\|_{1, q} \leq\|\varphi\|_{0, q^{\circ}}
\end{gathered}
$$

We have

$$
\begin{aligned}
\left(\left(u-u_{h}\right)_{x}, \varphi\right)= & A\left(t ; u-u_{h}, \psi\right)=A\left(t ; u-u_{h}, \psi-R_{h} \psi\right) \\
& +A\left(t ; u-u_{h}, R_{h} \psi-I_{h}^{*} R_{h} \psi\right) \\
& -\int_{0}^{t} B\left(t, s ;\left(u-u_{h}\right)(s), I_{h}^{*} R_{h} \psi\right) d s \\
& -\left(\left(u-u_{h}\right)_{t}, I_{h}^{*} R_{h} \psi\right) \\
= & I_{1}+I_{2}+I_{3}+I_{4}, \\
\left|I_{1}\right| \leq & \left|A\left(t ; u-R_{h} u, \psi\right)\right| \leq C\left\|u-R_{h} u\right\|_{1, p}\|\psi\|_{1, q} \\
\leq & C h\|u\|_{2, p}\|\psi\|_{1, q}
\end{aligned}
$$

By Lemma 4

$$
\begin{gathered}
\left|I_{2}\right| \leq A\left(t ; u-u_{h}, R_{h} \psi-I_{h}^{*} R_{h} \psi\right) \\
\leq C h\left(\left|u-u_{h}\right|_{1, p}+|u|_{2, p}\right)\|\psi\|_{1, q^{\prime}} \\
\left|I_{3}\right| \leq \int_{0}^{t}\left\|u-u_{h}\right\|_{1, p} d s\|\psi\|_{1, q^{\prime}} \\
\left|I_{4}\right| \leq\left(\left\|u-u_{h}\right\|\right)\|\psi\| \leq \operatorname{Ch}^{2}\left(\left\|u_{0}\right\|_{2}+\int_{0}^{t}\left\|u_{t}\right\|_{2} d s\right)\|\psi\|_{1, q^{\prime}}
\end{gathered}
$$

where we have used the fact $\|\psi\| \leq\|\psi\|_{1, r}, r>1$. Combining these estimates, we get

$$
\begin{aligned}
\left|\left(\left(u-u_{h}\right)_{x}, \varphi\right)\right| \leq & C h\left(\left\|u_{0}\right\|_{2}+\|u\|_{2, p}+\int_{0}^{t}\left\|u_{t}\right\|_{2} d s\right)\|\psi\|_{1, q^{\prime}} \\
\left\|\left(u-u_{h}\right)_{x}\right\|_{0, p}= & \sup \frac{\left(\left(u-u_{h}\right)_{x}, \varphi\right)}{\|\varphi\|_{0, q}} \\
\leq & C h\left|u-u_{h}\right|_{1, p} \\
& +\operatorname{Ch}\left(\left\|u_{0}\right\|_{2}+\|u\|_{2, p}+\int_{0}^{t}\left\|u_{t}\right\|_{2} d s\right) .
\end{aligned}
$$

Hence using the Poincaré inequality, we have for $h$ sufficiently small

$$
\left\|u-u_{h}\right\|_{1, p} \leq C h\left(\left\|u_{0}\right\|_{2}+\|u\|_{2, p}+\int_{0}^{t}\left\|u_{t}\right\|_{2} d s\right) .
$$

We compare the relationship between covolume solution and the Galerkin finite element solution.

Corollary 14. Let $\widetilde{u}_{h}$ be the finite element solution to (2); that is,

$$
\begin{gathered}
\left(\widetilde{u}_{h t}, v_{h}\right)+A\left(t ; \tilde{u}_{h}, v_{h}\right) \\
+\int_{0}^{t} B\left(t, s ; \widetilde{u}_{h}(s), v_{h}\right) d s=\left(f, v_{h}\right), \quad v_{h} \in S_{h}, \\
\tilde{u}_{h}(0)=R_{h} u_{0} .
\end{gathered}
$$

For h sufficiently small, we have

$$
\begin{aligned}
& \left\|\left(\widetilde{u}_{h}-u_{h}\right)\right\|_{1, p} \\
& \quad \leq C\left(\begin{array}{c}
h\left\|u-u_{h}\right\|_{1, p}+\left\|\left(u-u_{h}\right)_{t}\right\|+\left\|\left(\widetilde{u}_{h}-u\right)_{t}\right\| \\
+\int_{0}^{t}\left(\left\|\left(u-u_{h}\right)(s)\right\|_{1, p}+\left\|\left(u-\widetilde{u}_{h}\right)(s)\right\|_{1, p}\right) d s
\end{array}\right) \\
& \quad \leq C(u) h .
\end{aligned}
$$

Proof. By (2) and (67),

$$
\begin{aligned}
& \left(\left(\widetilde{u}_{h}-u\right)_{t}, v_{h}\right)+A\left(t ; \widetilde{u}_{h}-u, v_{h}\right) \\
& \quad+\int_{0}^{t} B\left(t, s ;\left(\widetilde{u}_{h}-u\right)(s), v_{h}\right) d s=0, \quad v_{h} \in S_{h} .
\end{aligned}
$$

Consider the following auxiliary problem. For any $\operatorname{such} \varphi$, let $\psi$ be the solution of the following:

$$
\begin{gathered}
A(t)^{*} \psi=-\varphi_{x}, \quad \text { in } \Omega, \\
\psi=0, \quad \text { on } \partial \Omega,
\end{gathered}
$$


with

$$
\begin{aligned}
& \|\psi\|_{1, q} \leq\|\varphi\|_{0, q}, \\
\left(\left(\widetilde{u}_{h}-u_{h}\right)_{x}, \varphi\right) & \\
= & A\left(t ; \widetilde{u}_{h}-u_{h}, \psi\right) \\
= & A\left(t ; \widetilde{u}_{h}-u_{h}, \psi-R_{h} \psi\right)+A\left(t ; u-u_{h}, R_{h} \psi\right) \\
& -A\left(t ; u-u_{h}, I_{h}^{*} R_{h} \psi\right)-\left(\left(u-u_{h}\right)_{t}, I_{h}^{*} R_{h} \psi\right) \\
& -\int_{0}^{t} B\left(t, s ;\left(u-u_{h}\right)(s), I_{h}^{*} R_{h} \psi\right) d s \\
& +A\left(t ; \widetilde{u}_{h}-u, R_{h} \psi\right) \\
= & {\left[A\left(t ; u-u_{h}, R_{h} \psi\right)-A\left(t ; u-u_{h}, I_{h}^{*} R_{h} \psi\right)\right] } \\
& -\left(\left(u-u_{h}\right)_{t}, I_{h}^{*} R_{h} \psi\right)-\left(\left(\widetilde{u}_{h}-u\right)_{t}, R_{h} \psi\right) \\
& -\int_{0}^{t} B\left(t, s ;\left(u-u_{h}\right)(s), I_{h}^{*} R_{h} \psi\right) d s \\
& -\int_{0}^{t} B\left(t, s ;\left(\widetilde{u}_{h}-u\right)(s), R_{h} \psi\right) d s \\
= & I_{1}+I_{2}+I_{3} .
\end{aligned}
$$

On the other hand,

$$
\begin{gathered}
\left|I_{1}\right| \leq C h\left\|u-u_{h}\right\|_{1, p}\|\psi\|_{1, q^{\prime}} \\
\left|I_{2}\right| \leq C\left(\left\|\left(u-u_{h}\right)_{t}\right\|+\left\|\left(\widetilde{u}_{h}-u\right)_{t}\right\|\right)\|\psi\| \\
\leq C\left(\left\|\left(u-u_{h}\right)_{t}\right\|+\left\|\left(\widetilde{u}_{h}-u\right)_{t}\right\|\right)\|\psi\|_{1, q^{\prime}}
\end{gathered}
$$

where we have used the fact $\|\psi\| \leq\|\psi\|_{1, r}, r>1$

$$
\begin{aligned}
& \left|I_{3}\right| \leq \int_{0}^{t}\left(\left\|\left(u-u_{h}\right)(s)\right\|_{1, p}+\left\|\left(u-\widetilde{u}_{h}\right)(s)\right\|_{1, p}\right) d s\|\psi\|_{1, q} \\
& \left\|\left(\widetilde{u}_{h}-u_{h}\right)_{x}\right\|_{0, p} \\
& =\sup _{\varphi \in \mathscr{C}_{0}^{\infty}} \frac{\left(\left(\widetilde{u}_{h}-u_{h}\right)_{x}, \varphi\right)}{\|\varphi\|_{0, q}} \\
& \quad \leq C\left(\begin{array}{c}
h\left\|u-u_{h}\right\|_{1, p}+\left\|\left(u-u_{h}\right)_{t}\right\|+\left\|\left(\widetilde{u}_{h}-u\right)_{t}\right\| \\
+\int_{0}^{t}\left(\left\|\left(u-u_{h}\right)(s)\right\|_{1, p}+\left\|\left(u-\widetilde{u}_{h}\right)(s)\right\|_{1, p}\right) d s
\end{array}\right) .
\end{aligned}
$$

We deduce the result from the known finite element estimates.

Remark 15. In order to estimate $\left\|\left(u-u_{h}\right)_{t}\right\|$, by differentiating (42) with respect to $t$, we obtain

$$
\begin{aligned}
\left(\theta_{t t}, I_{h}^{*} v_{h}\right)+A\left(t ; \theta_{t}, I_{h}^{*} v_{h}\right)+A_{t}\left(t ; \theta_{t}, I_{h}^{*} v_{h}\right) & \\
& +B\left(t, t ; \theta, I_{h}^{*} v_{h}\right)+\int_{0}^{t} B_{t}\left(t, s ; \theta(s), I_{h}^{*} v_{h}\right) d s \\
= & -\left(\rho_{t t}, I_{h}^{*} v_{h}\right) .
\end{aligned}
$$

Setting $v_{h}=\theta_{t}$, we obtain

$$
\begin{aligned}
& \frac{1}{2} \frac{d}{d t}\left\|\theta_{t}\right\|\left\|^{2}+c\right\| \theta_{t}\left\|_{1}^{2} \leq\right\| \rho_{t t}\|\| \theta_{t} \| \\
& +\frac{1}{2} c\left\|\theta_{t}\right\|_{1}^{2}+C\|\theta\|_{1}^{2}+\int_{0}^{t}\|\theta\|_{1}^{2} d s \\
& \leq\left\|\rho_{t t}\right\|\left\|\theta_{t}\right\|+\frac{1}{2} c\left\|\theta_{t}\right\|_{1}^{2}+C \int_{0}^{t}\left\|\theta_{t}\right\|_{1}^{2} d s .
\end{aligned}
$$

Using kickback argument, integrating and applying Gronwall's lemma, we deduce

$$
\begin{aligned}
\left\|\theta_{t}\right\| & \leq C\left(\left\|\theta_{t}(0)\right\|+\int_{0}^{t}\left\|\rho_{t t}\right\|_{1} d s\right) \\
& \leq C h^{2}\left(\left\|u_{0}\right\|_{2}+\left\|u_{1}\right\|_{2}+\int_{0}^{t}\left\|u_{t t}\right\|_{2} d s\right) .
\end{aligned}
$$

\section{The Lumped Mass Finite Volume Element Method}

In this section, we restrict our study to the $2 \mathrm{D}$ case. A simple way to define the lumped mass scheme [21] is to replace the mass matrix $M_{h}$ in (14) by the diagonal matrix $\bar{M}_{h}$ obtained by taking for its diagonal elements the numbers $\bar{M}_{h i i}=$ $\sum_{j=1}^{N_{h}} M_{h i j}$ or by lumping all masses in one row into the diagonal entry. This makes the inversion of the matrix in front of $\alpha^{\prime}(t)$ a triviality. We will therefore study the matrix problem

$$
\bar{M}_{h} \alpha^{\prime}(t)+A_{h}(t) \alpha(t)+\int_{0}^{t} B_{h}(t, s) \alpha(s) d s=F_{h}(t) .
$$

We know that the lumped mass method defined by (77) above is equivalent to

$$
\begin{gathered}
\left(I_{h}^{*} u_{h t}, I_{h}^{*} v_{h}\right)+A\left(t ; u_{h}, I_{h}^{*} v_{h}\right)+\int_{0}^{t} B\left(t, s ; u_{h}(s), I_{h}^{*} v_{h}\right) d s \\
=\left(f, I_{h}^{*} v_{h}\right), \quad v_{h} \in S_{h} .
\end{gathered}
$$

Our alternative interpretation of this procedure will be to think of (77) as being obtained by evaluating the first term in (78) by numerical quadrature. Let $K$ be a triangle of the triangulation $T_{h}$, let $x_{j}, j=1,2,3$, be its vertices, and consider the quadrature formula

$$
Q_{K, h}(f)=\frac{1}{3} \text { area } K \sum_{j=1}^{3} f\left(x_{j}\right) \simeq \int_{K} f d x .
$$

We may then define the associated bilinear form in $S_{h} \times$ $S_{h}^{*}$, using the quadrature scheme, by the following:

$$
\begin{array}{r}
\left(v_{h}, \eta_{h}\right)_{h}=\sum_{K \in T_{h}} Q_{K, h}\left(v_{h} \eta_{h}\right)=\sum_{x_{i} \in N_{h}^{a}} v_{h}\left(x_{i}\right) \eta_{h}\left(x_{i}\right)\left|V_{x_{i}}\right|, \\
\forall v_{h} \in S_{h}, \quad \eta_{h} \in S_{h}^{*} .
\end{array}
$$


We note that $\left\|v_{h}\right\|_{h}^{2}=\left(v_{h}, I_{h}^{*} v_{h}\right)_{h}$ is a norm in $S_{h}$ which is equivalent to the $L^{2}$-norm uniformly in $h$; that is, there exist two positive constants $C_{1}$ and $C_{2}$ such that for all $v_{h} \in S_{h}$, we have

$$
C_{0}\left\|v_{h}\right\| \leq\left\|v_{h}\right\|_{h} \leq C_{1}\left\|v_{h}\right\|, \quad \forall v_{h} \in S_{h} .
$$

We note that the aforementioned definition $\left(v_{h}, \eta_{h}\right)_{h}$ may be used also for $\eta_{h} \in S_{h}$ and that $\left(v_{h}, w_{h}\right)_{h}=\left(v_{h}, I_{h}^{*} w_{h}\right)_{h}$ for $v_{h}, w_{h} \in S_{h}$.

The lumped mass method defined by (78) is equivalent to

$$
\begin{gathered}
\left(u_{h t}, I_{h}^{*} v_{h}\right)_{h}+A\left(t ; u_{h}, I_{h}^{*} v_{h}\right)+\int_{0}^{t} B\left(t, s ; u_{h}(s), I_{h}^{*} v_{h}\right) d s \\
=\left(f, I_{h}^{*} v_{h}\right), \quad v_{h} \in S_{h} .
\end{gathered}
$$

We introduce the quadrature error

$$
\varepsilon_{h}\left(v_{h}, w_{h}\right)=\left(v_{h}, w_{h}\right)_{h}-\left(v_{h}, w_{h}\right),
$$

Lemma 16 (see [21]). Let $v_{h}, w_{h} \in S_{h}$. Then

$$
\left|\varepsilon_{h}\left(v_{h}, w_{h}\right)\right| \leq C h^{2}\left\|\nabla v_{h}\right\|\left\|\nabla w_{h}\right\| .
$$

Theorem 17. Let $u_{h}$ and $u$ be the solutions of (82) and (2), respectively, and assume $u_{h}(0)=R_{h} u_{0}$. Then we have for the error in the lumped mass semidiscrete method, for $t \geq 0$, the following:

$$
\left\|u_{h}(t)-u(t)\right\| \leq C h^{2}\left(\left\|u_{0}\right\|_{2}+\|u\|_{2}+\int_{0}^{t}\left\|u_{t}\right\|_{2} d s\right) .
$$

Proof. In order to estimate $\|\theta\|$, we write

$$
\begin{aligned}
\left(\theta_{t}, I_{h}^{*} v_{h}\right)_{h}+A\left(t ; \theta, I_{h}^{*} v_{h}\right)+\int_{0}^{t} B\left(t, s ; \theta(s), I_{h}^{*} v_{h}\right) d s \\
=\left(u_{h t}, I_{h}^{*} v_{h}\right)_{h}+A\left(t ; u_{h}, I_{h}^{*} v_{h}\right) \\
\quad+\int_{0}^{t} B\left(t, s ; u_{h}(s), I_{h}^{*} v_{h}\right) d s \\
\quad-\left(\left(V_{h} u\right)_{t}, I_{h}^{*} v_{h}\right)_{h}-A\left(t ; V_{h} u, I_{h}^{*} v_{h}\right) \\
\quad-\int_{0}^{t} B\left(t, s ; V_{h} u(s), I_{h}^{*} v_{h}\right) d s \\
=\left(f, I_{h}^{*} v_{h}\right)-\left(\left(V_{h} u\right)_{t}, I_{h}^{*} v_{h}\right)_{h}-A\left(t ; u, I_{h}^{*} v_{h}\right) \\
\quad-\int_{0}^{t} B\left(t, s ; u(s), I_{h}^{*} v_{h}\right) \\
=\left(u_{t}, I_{h}^{*} v_{h}\right)-\left(\left(V_{h} u\right)_{t}, I_{h}^{*} v_{h}\right)_{h} \\
=-\left(\rho_{t}, I_{h}^{*} v_{h}\right)-\left(\left(V_{h} u\right)_{t}, I_{h}^{*} v_{h}\right)_{h}+\left(\left(V_{h} u\right)_{t}, I_{h}^{*} v_{h}\right) .
\end{aligned}
$$

We rewrite

$$
\begin{aligned}
&\left(\left(V_{h} u\right)_{t}, I_{h}^{*} v_{h}\right)_{h}-\left(\left(V_{h} u\right)_{t}, I_{h}^{*} v_{h}\right) \\
&=\left(\left(V_{h} u\right)_{t}, I_{h}^{*} v_{h}\right)_{h}-\left(\left(V_{h} u\right)_{t}, v_{h}\right) \\
&+\left(\left(V_{h} u\right)_{t}, v_{h}\right)-\left(\left(V_{h} u\right)_{t}, I_{h}^{*} v_{h}\right) \\
&= \varepsilon_{h}\left(\left(V_{h} u\right)_{t}, v_{h}\right)+\left(\left(V_{h} u\right)_{t}, v_{h}\right)-\left(\left(V_{h} u\right)_{t}, I_{h}^{*} v_{h}\right) . \\
&\left(\theta_{t}, I_{h}^{*} v_{h}\right)_{h}+A\left(t ; \theta, I_{h}^{*} v_{h}\right)+\int_{0}^{t} B\left(t, s ; \theta(s), I_{h}^{*} v_{h}\right) d s \\
&=-\left(\rho_{t}, I_{h}^{*} v_{h}\right)+\varepsilon_{h}\left(\left(V_{h} u\right)_{t}, v_{h}\right) \\
&+\left(\left(V_{h} u\right)_{t}, v_{h}\right)-\left(\left(V_{h} u\right)_{t}, I_{h}^{*} v_{h}\right) .
\end{aligned}
$$

Setting $v_{h}=\theta$ in (87), we obtain

$$
\begin{aligned}
\frac{1}{2} \frac{d}{d t}\|\theta\|_{h}^{2}+c_{0}\|\theta\|_{1}^{2} & \\
\leq & \left\|\rho_{t}\right\|\|\theta\|+\frac{1}{2} c_{0}\|\theta\|_{1}^{2}+C \int_{0}^{t}\|\theta\|_{1}^{2} d s \\
& +\varepsilon_{h}\left(\left(V_{h} u\right)_{t}, \theta\right)+\left(\left(V_{h} u\right)_{t}, \theta\right)-\left(\left(V_{h} u\right)_{t}, I_{h}^{*} \theta\right) .
\end{aligned}
$$

Using Lemma 16 and the inverse estimate, we get

$$
\begin{aligned}
\left|\varepsilon_{h}\left(V_{h} u_{t}, \theta\right)\right| & \leq C h^{2}\left\|\nabla\left(V_{h} u\right)_{t}\right\|\|\nabla \theta\| \\
& \leq C h^{2}\left\|\nabla u_{t}\right\|\|\nabla \theta\| \\
& \leq C h\left\|\nabla u_{t}\right\|\|\theta\|,
\end{aligned}
$$

we have

$$
\left|\left(\left(V_{h} u\right)_{t}, \theta\right)-\left(\left(V_{h} u\right)_{t}, I_{h}^{*} \theta\right)\right| \leq C h\left\|\nabla u_{t}\right\|\|\theta\| .
$$

Using Young's inequality and Gronwall's lemma to eliminate $\|\theta\|_{1}$ on the right-hand side and using integration in $t$, we get the result.

$$
\frac{1}{2} \frac{d}{d t}\|\theta\|_{h}^{2}+c_{0}\|\theta\| \leq\left\|\rho_{t}\right\|\|\theta\|+C h\left\|\nabla u_{t}\right\|\|\theta\| .
$$

Using Young's inequality to eliminate $\|\theta\|$ on the right hand side it becomes.

Using integration in $t$, we get the result.

We will now show that the $H^{1}$-norm error bound of theorem remains valid for the lumped mass method (82).

Theorem 18. Let $u_{h}$ and $u$ be the solutions of (82) and (2), respectively, and assume

$$
u_{h}(0)=R_{h} u_{0}, \quad\left\|u_{1 h}(0)-u_{1}\right\| \leq C h^{2}\left\|u_{1}\right\|_{2} .
$$

Then, we have for the error in the lumped mass semidiscrete method, for $t \geq 0$, the following:

$$
\left\|u_{h}(t)-u(t)\right\|_{1} \leq C h^{2}\left(\left\|u_{0}\right\|_{2}+\left\|u_{1}\right\|_{2}+\int_{0}^{t}\left\|u_{t t}\right\|_{2} d s\right) .
$$


Proof. Setting $v_{h}=\theta_{t}$ in (87), we obtain

$$
\begin{aligned}
&\left\|\theta_{t}\right\|_{h}^{2}+\frac{1}{2} \frac{d}{d t} A\left(t ; \theta, I_{h}^{*} \theta\right) \\
&= \frac{1}{2} A_{t}\left(t ; \theta, I_{h}^{*} \theta\right)+\frac{1}{2}\left[A\left(t ; \theta_{t}, I_{h}^{*} \theta\right)-A\left(t ; \theta, I_{h}^{*} \theta_{t}\right)\right] \\
& B\left(t, t ; \theta(t), I_{h}^{*} \theta(t)\right)+\int_{0}^{t} B_{t}\left(t, s ; \theta(s), I_{h}^{*} \theta(t)\right) d s \\
& \quad-\frac{d}{d t} \int_{0}^{t} B\left(t, s ; \theta(s), I_{h}^{*} \theta(t)\right) d s-\left(\rho_{t}, I_{h}^{*} \theta_{t}\right) \\
& \quad-\varepsilon_{h}\left(\left(V_{h} u\right)_{t}, \theta_{t}\right)+\left(\left(V_{h} u\right)_{t}, \theta\right)-\left(\left(V_{h} u\right)_{t}, I_{h}^{*} \theta\right) .
\end{aligned}
$$

It follows, thus, that using integration in $t$ and Gronwall's lemma, we have

$$
\begin{gathered}
\int_{0}^{t}\left\|\theta_{t}\right\|_{h}^{2}+\|\theta\|_{1}^{2} \leq C\|\nabla \theta(0)\|^{2}+C \int_{0}^{t}\left\|\rho_{t}\right\|\left\|\theta_{t}\right\| d s \\
+C h^{2} \int_{0}^{t}\left\|u_{t}\right\|_{1}^{2} d s .
\end{gathered}
$$

\section{Full Discretization}

Let $\bar{\partial} U^{n}=\left(U^{n}-U^{n-1}\right) / k$ be the backward difference quotient of $U^{n}$; assume that $A_{h}=P_{h} A$ is a discrete analogue of $A$ ( similarly $B_{h}=P_{h} B$ ), where $P_{h}: L^{2}(\Omega) \rightarrow S_{h}^{*}$ the $L^{2}$ projection is defined by

$$
\left(P_{h} v, I_{h}^{*} v_{h}\right)=\left(v, I_{h}^{*} v_{h}\right), \quad v \in L^{2}(\Omega), v_{h} \in S_{h} .
$$

In order to define fully discrete approximation of (11), we discretize the time by taking $t_{n}=n k, k>0, n=1,2, \ldots$ and use the numerical quadrature

$$
\int_{0}^{t_{n-1 / 2}} g(s) d s \approx \sum_{k=1}^{n} \omega_{n, k} g\left(t_{k-1 / 2}\right), \quad t_{n-1 / 2}=\left(n-\frac{1}{2}\right) k .
$$

Here $\left\{\omega_{n, k}\right\}$ are the integration weights and we assume that the following error estimate is valid:

$$
\begin{aligned}
q^{n}(g) & =\int_{0}^{t_{n-1 / 2}} g(s) d s-\sum_{k=1}^{n} \omega_{n, k} g\left(t_{k-1 / 2}\right) \\
& \leq C k^{2} \int_{0}^{t_{n}}\left(\left|g^{\prime}\right|+\left|g^{\prime \prime}\right|\right) d s .
\end{aligned}
$$

Now, define our complete discrete FVE approximation of (11) by the following: find $U^{n} \in S_{h}$ for $n=1,2, \ldots$, such that for all $v_{h} \in S_{h}$

$$
\begin{gathered}
\left(\bar{\partial} U^{n}, I_{h}^{*} v_{h}\right)+A\left(t_{n-1 / 2} ; U^{n-1 / 2}, I_{h}^{*} v_{h}\right) \\
+\sum_{k=1}^{n} \omega_{n, k} B\left(t_{n-1 / 2}, t_{k-1 / 2}, U^{k-1 / 2}, I_{h}^{*} v_{h}\right) \\
=\left(f^{n-1 / 2}, I_{h}^{*} v_{h}\right), \\
U^{0} \text { in } S_{h},
\end{gathered}
$$

where $U^{n-1 / 2}=\left(U^{n}+U^{n-1}\right) / 2$.
Theorem 19. Let $u(t)$ and $U^{n}$ be the solutions of problem (2) and its complete discrete scheme (99), respectively. Then for any $T>0$ there exists a positive constant $C=C(T)>0$, independent of $h$, such that for $0<t_{n} \leq T$

$$
\begin{aligned}
\| u\left(t_{n}\right)- & U^{n} \| \\
\leq & C h^{2}\left(\left\|u_{0}\right\|_{2}+\int_{0}^{t_{n}}\left\|u_{t}\right\|_{2} d s\right) \\
& +C k^{2}\left(\int_{0}^{t_{n}}\left(\|u\|_{2}+\left\|u_{t}\right\|_{2}+\left\|u_{t t}\right\|_{2}+\left\|u_{t t t}\right\|\right) d s\right) .
\end{aligned}
$$

Proof. Let us split the error into two parts: $u\left(t_{n}\right)-U^{n}=\rho^{n}+$ $\theta^{n}$, where $\rho^{n}=u\left(t_{n}\right)-V_{h} u\left(t_{n}\right)$ and $\theta^{n}=V_{h} u\left(t_{n}\right)-U^{n}$, and let $W=V_{h} u(t) \in S_{h}$ be the Ritz-Volterra projection of $u$. Then from (2) and (99) we have for all $v_{h} \in S_{h}$ the following:

$$
\begin{aligned}
& \left(\bar{\partial} \theta^{n}, I_{h}^{*} v_{h}\right)+A\left(t_{n-1 / 2} ; \theta^{n-1 / 2}, I_{h}^{*} v_{h}\right) \\
& \quad+\sum_{k=1}^{n} \omega_{n, k} B\left(t_{n-1 / 2}, t_{k-1 / 2}, \theta^{k-1 / 2}, I_{h}^{*} v_{h}\right) \\
& =-\left(r_{n}, I_{h}^{*} v_{h}\right), \quad \forall v_{h} \in S_{h},
\end{aligned}
$$

where

$$
\begin{aligned}
r_{n}= & r_{n}^{1}+r_{n}^{2}+r_{n}^{3}+r_{n}^{4}, \\
r_{n}^{1}= & \bar{\partial} \rho^{n} \\
r_{n}^{2}= & \bar{\partial} u\left(t_{n}\right)-u_{t}\left(t_{n-1 / 2}\right), \\
r_{n}^{3}= & A\left(t_{n-1 / 2} ; \frac{\left(u\left(t_{n}\right)+u\left(t_{n-1}\right)\right)}{2}-u\left(t_{n-1 / 2}\right)\right), \\
r_{n}^{4}= & q^{n}\left(B_{h} W\right) \\
= & \sum_{k=1}^{n} \omega_{n, k} B_{h}\left(t_{n-1 / 2}, t_{k-1 / 2}, W^{k-1 / 2}, I_{h}^{*} v_{h}\right) \\
& -\int_{0}^{t_{n-1 / 2}} B\left(t_{n}, s, W(s), I_{h}^{*} v_{h}\right) d s .
\end{aligned}
$$

In fact, by Taylor expansion,

$$
\begin{aligned}
u^{n+1}= & u^{n}+k u^{\prime}\left(t_{n}\right)+\int_{t_{n}}^{t_{n+1}} u^{\prime \prime}(s)\left(t_{n+1}-s\right) d s \\
= & u^{n}+k u^{\prime}\left(t_{n}\right)+\frac{k^{2}}{2} u^{\prime \prime}\left(t_{n}\right)+\frac{k^{3}}{6} u^{(3)}\left(t_{n}\right) \\
& +\frac{1}{6} \int_{t_{n}}^{t_{n+1}} u^{(4)}(s)\left(t_{n+1}-s\right)^{3} d s,
\end{aligned}
$$


we have

$$
\begin{aligned}
\left\|r_{n}^{1}\right\| & =\left\|\bar{\partial} \rho^{n}\right\| \leq \frac{1}{k} \int_{t_{n-1}}^{t_{n}}\left\|\rho_{t}\right\| d s \leq C \frac{h^{2}}{k} \int_{t_{n-1}}^{t_{n}}\left\|u_{t t}\right\|_{2} d s, \\
\left\|r_{n}^{2}\right\| & =\left\|\bar{\partial} u\left(t_{n}\right)-u_{t}\left(t_{n-1 / 2}\right)\right\| \\
& =\frac{1}{k}\left\|\int_{t_{n-1}}^{t_{n}}\left(u_{t}(s)-u_{t}\left(t_{n-1 / 2}\right)\right) d s\right\| \\
& \leq C k \int_{t_{n-1}}^{t_{n}}\left\|u^{(3)}(s)\right\| d s, \\
\left\|r_{n}^{3}\right\| & =\left\|A\left(t_{n-1 / 2} ; \frac{u\left(t_{n}\right)+u\left(t_{n-1}\right)}{2}-u\left(t_{n-1 / 2}\right), I_{h}^{*} v_{h}\right)\right\| \\
& \leq C k \int_{t_{n-1}}^{t_{n}}\left\|A u_{t t}(s)\right\| d s \leq C k \int_{t_{n-1}}^{t_{n}}\left\|u_{t t}\right\|_{2} d s .
\end{aligned}
$$

In addition, the quadrature error satisfies

$$
\begin{aligned}
\left\|r_{n}^{4}\right\|= & q^{n-1 / 2}\left(B_{h} W\right) \\
= & \sum_{k=1}^{n} \omega_{n, k} B\left(t_{n-1 / 2}, t_{k-1 / 2}, W^{k-1 / 2}, I_{h}^{*} v_{h}\right) \\
& -\int_{0}^{t_{n-1 / 2}} B\left(t_{n}, s, W(s), I_{h}^{*} v_{h}\right) d s \\
\leq & C k^{2} \int_{0}^{t_{n}}\left\|\left(B_{h} W\right)_{s s}\right\| d s \\
\leq & C k^{2} \int_{0}^{t_{n}}\left(\|u\|_{2}+\left\|u_{t}\right\|_{2}+\left\|u_{t t}\right\|_{2}\right) d s . \\
k \sum_{n=1}^{N}\left\|r_{n}\right\| \leq & C h^{2} \int_{0}^{t_{n}}\left\|u_{t t}\right\|_{2} d s \\
& +C k^{2} \int_{0}^{t_{n}}\left(\|u\|_{2}+\left\|u_{t}\right\|_{2}+\left\|u_{t t}\right\|_{2}+\left\|u^{(3)}\right\|\right) d s .
\end{aligned}
$$

Taking $v_{h}=\theta^{n-1 / 2}$ in (101) and noting that $\left(\bar{\partial} \theta^{n}, I_{h}^{*} \theta^{n-1 / 2}\right)=$ $(1 / 2) \bar{\partial}\|\| \theta^{n} \|\left.\right|^{2}$, there is

$$
\begin{aligned}
\left.\left\|\theta^{n}\right\|\right|^{2}-\left.\left\|\theta^{n-1}\right\|\right|^{2}+2 k c\|\| \theta^{n-1 / 2} \|_{1}^{2} \\
\leq C k^{2} \sum_{k=1}^{n}\left\|\theta^{k-1 / 2}\right\|_{1}\left\|\theta^{n-1 / 2}\right\|_{1}+C k\left\|r_{n}\right\|\left\|\theta^{n-1 / 2}\right\| \\
\quad \leq k c\left\|\theta^{n-1 / 2}\right\|_{1}^{2}+C k^{2} \sum_{k=1}^{n}\left\|\theta^{k-1 / 2}\right\|_{1}^{2}+C k\left\|r_{n}\right\|\left\|\theta^{n-1 / 2}\right\| .
\end{aligned}
$$

Summing from $n=1$ to $N$ and then, after cancelling the common factor and using Gronwall's lemma, we obtain

$$
\left.\left\|\theta^{N}\right\|\right|^{2} \leq\left. C\left\|\theta^{0}\right\|\right|^{2}+C k \sum_{k=1}^{N}\left\|r_{n}\right\|\left(\left\|\theta^{k}\right\|+\left\|\theta^{k-1 / 2}\right\|\right),
$$

and then

$$
\left\|\left|\theta ^ { N } \left\|\left|\leq C\left\|\left|\theta^{0}\left\|\mid+C k \sum_{n=1}^{N}\right\| r_{n} \|,\right.\right.\right.\right.\right.\right.
$$

the theorem follows from the estimates of $\rho^{n}$ and $r^{n}$.

\section{References}

[1] Z. Q. Cai, "On the finite volume element method," Numerische Mathematik, vol. 58, no. 7, pp. 713-735, 1991.

[2] S. H. Chou and D. Y. Kwak, "Analysis and convergence of a MAC-like scheme for the generalized Stokes problem," Numerical Methods for Partial Differential Equations, vol. 13, no. 2, pp. 147-162, 1997.

[3] P. Chatzipantelidis, "A finite volume method based on the Crouzeix-Raviart element for elliptic PDE's in two dimensions," Numerische Mathematik, vol. 82, no. 3, pp. 409-432, 1999.

[4] P. Chatzipantelidis, "Finite volume methods for elliptic PDE's: a new approach," Mathematical Modelling and Numerical Analysis, vol. 36, no. 2, pp. 307-324, 2002.

[5] P. Chatzipantelidis, R. D. Lazarov, and V. Thomée, "Error estimates for a finite volume element method for parabolic equations in convex polygonal domains," Numerical Methods for Partial Differential Equations, vol. 20, no. 5, pp. 650-674, 2004.

[6] R. E. Ewing, R. D. Lazarov, and Y. Lin, "Finite volume element approximations of nonlocal in time one-dimensional flows in porous media," Computing, vol. 64, no. 2, pp. 157-182, 2000.

[7] R. Ewing, R. Lazarov, and Y. Lin, "Finite volume element approximations of nonlocal reactive flows in porous media," Numerical Methods for Partial Differential Equations, vol. 16, no. 3, pp. 285-311, 2000.

[8] R. E. Ewing, T. Lin, and Y. Lin, "On the accuracy of the finite volume element method based on piecewise linear polynomials," SIAM Journal on Numerical Analysis, vol. 39, no. 6, pp. 1865$1888,2002$.

[9] R. K. Sinha and J. Geiser, "Error estimates for finite volume element methods for convection-diffusion-reaction equations," Applied Numerical Mathematics, vol. 57, no. 1, pp. 59-72, 2007.

[10] S. H. Chou, "Analysis and convergence of a covolume method for the generalized Stokes problem," Mathematics of Computation, vol. 66, no. 217, pp. 85-104, 1997.

[11] M. Berggren, "A vertex-centered, dual discontinuous Galerkin method," Journal of Computational and Applied Mathematics, vol. 192, no. 1, pp. 175-181, 2006.

[12] S. H. Chou and D. Y. Kwak, "Multigrid algorithms for a vertexcentered covolume method for elliptic problems," Numerische Mathematik, vol. 90, no. 3, pp. 441-458, 2002.

[13] R. Eymard, T. Gallouët, and R. Herbin, Finite Volume Methods: Handbook of Numerical Analysis, North-Holland, Amsterdam, The Netherlands, 2000.

[14] V. R. Voller, Basic Control Volume Finite Element Methods for Fluids and Solids, World Scientific Publishing, 2009.

[15] J. Huang and S. Xi, "On the finite volume element method for general self-adjoint elliptic problems," SIAM Journal on Numerical Analysis, vol. 35, no. 5, pp. 1762-1774, 1998.

[16] X. Ma, S. Shu, and A. Zhou, "Symmetric finite volume discretizations for parabolic problems," Computer Methods in Applied Mechanics and Engineering, vol. 192, no. 39-40, pp. 4467-4485, 2003.

[17] R. D. Lazarov and S. Z. Tomov, "Adaptive finite volume element method for convection-diffusion-reaction problems in 3-D," in Scientific Computing and Applications, vol. 7 of Advances in Computation Theory and Practice, pp. 91-106, Nova Science Publishers, Huntington, NY, USA, 2001.

[18] I. D. Mishev, Finite volume and finite volume element methods for non-symmetric problems [Ph.D. thesis], Texas A\&M University, 1997, Technical Report ISC-96-04-MATH. 
[19] C. Chen and T. Shih, Finite Element Methods for Integrodifferential Equations, World Scientific Publishing, Singapore, 1998.

[20] Y. P. Lin, V. Thomée, and L. B. Wahlbin, "Ritz-Volterra projections to finite-element spaces and applications to integrodifferential and related equations," SIAM Journal on Numerical Analysis, vol. 28, no. 4, pp. 1047-1070, 1991.

[21] V. Thomée, Galerkin Finite Element Methods for Parabolic Problems, Springer Series in Computational Mathematics, Springer, New York, NY, USA, 2006. 


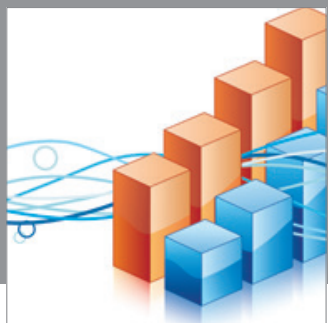

Advances in

Operations Research

mansans

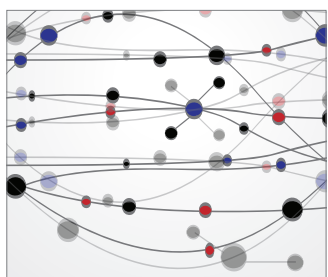

The Scientific World Journal
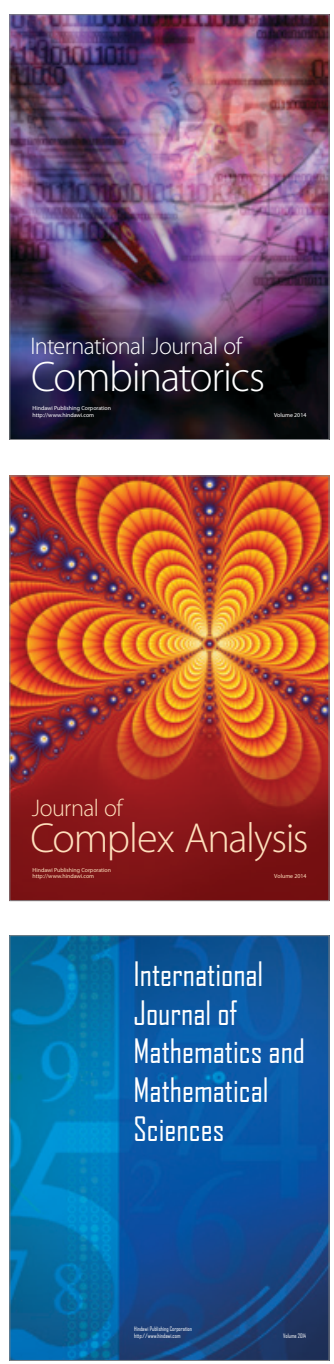
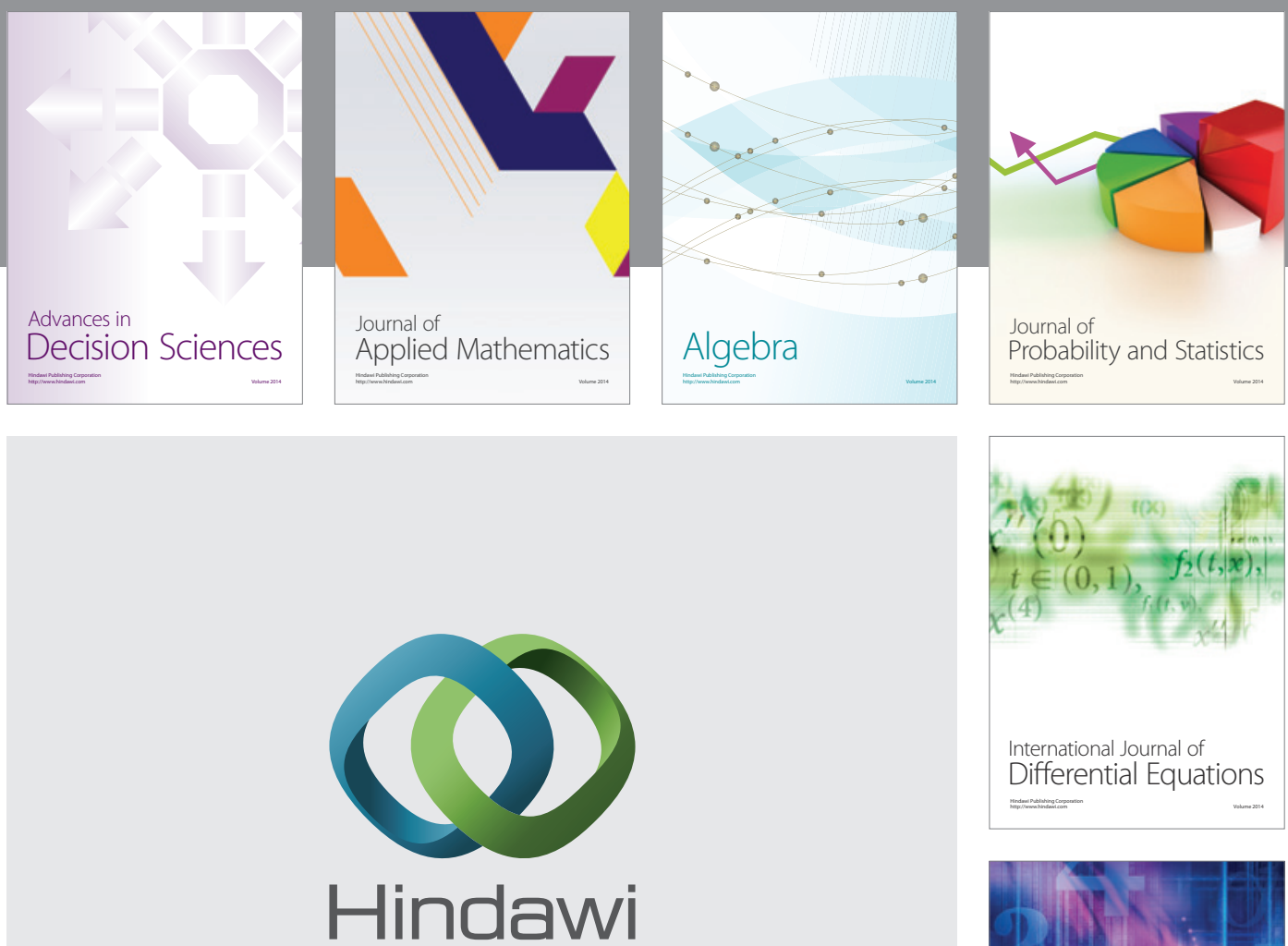

Submit your manuscripts at http://www.hindawi.com
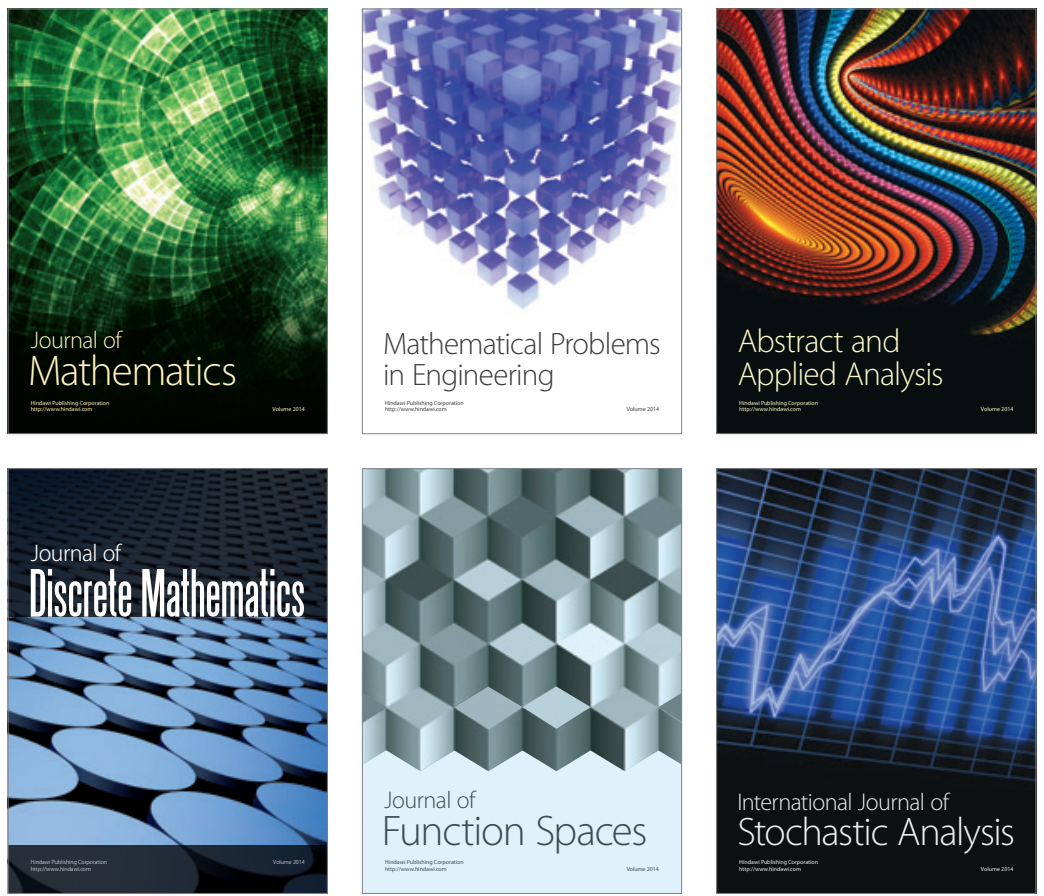

Journal of

Function Spaces

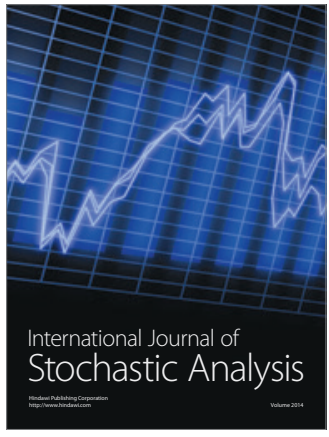

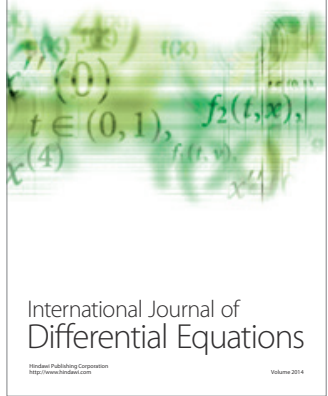
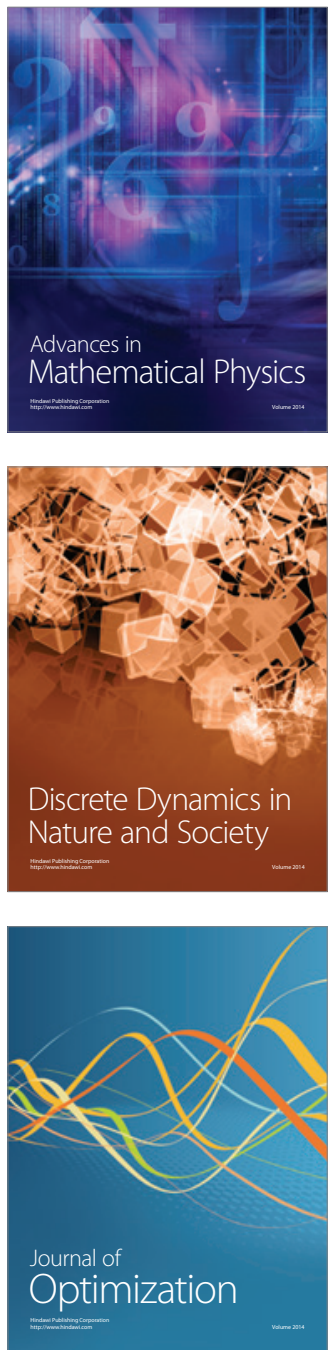